\title{
FOXP3 facilitates the invasion and metastasis of non-small cell lung cancer cells through regulating VEGF, EMT and the Notch1/Hes1 pathway
}

\author{
CHUN LI $^{1 *}$, HEFEI WANG ${ }^{2 *}$, HUI FANG ${ }^{1,3}$, CHENGYUAN HE $^{1}$, YIJIE PEI $^{1}$ and XIAODONG GAI ${ }^{1}$ \\ ${ }^{1}$ Department of Immunology, School of Basic Medical Sciences, Beihua University, Jilin, Jilin 132013; \\ ${ }^{2}$ Department of Gynecology, The Third Affiliated Hospital of Xiang Ya School of Medicine, Central South University, \\ Changsha, Hunan 410013; ${ }^{3}$ Department of Laboratory, Chifeng Clinical Medical School, \\ Inner Mongolia Medical University, Chifeng, Inner Mongolia 024000, P.R. China
}

Received September 10, 2020; Accepted March 8, 2021

DOI: $10.3892 /$ etm.2021.10390

\begin{abstract}
Forkhead box P3 (FOXP3) is a specific marker of regulatory $\mathrm{T}$ cells (Tregs) that is also expressed in tumour cells. Previous studies have revealed that FOXP3 can promote metastasis in several types of cancer, including non-small cell lung cancer (NSCLC); however, the underlying mechanism of FOXP3 remains unclear. The aim of the present study was to investigate the effect of FOXP3 on vascular endothelial growth factor (VEGF), epithelial-to-mesenchymal transition (EMT) and the Notch1/Hes1 pathway in NSCLC. After FOXP3 small interfering RNA (siRNAs) were transfected into A549 cells, the expression of FOXP3 mRNA and protein was determined by reverse transcription-quantitative PCR and western blotting. Cell migration and invasion were analyzed by Transwell assays. The concentrations of matrix metalloproteinase (MMP)-2, MMP-9 and VEGF in the cell supernatant were evaluated by ELISA. The expression of relevant proteins involved in EMT and Notch1/Hes1 pathway were assessed via western blotting. Additionally, the expression of FOXP3, CD31
\end{abstract}

Correspondence to: Professor Xiaodong Gai, Department of Immunology, School of Basic Medical Sciences, Beihua University, 3999 Binjiang East Road, Jilin, Jilin 132013, P.R. China

E-mail: bhdxgaixiaodong@126.com

${ }^{*}$ Contributed equally

Abbreviations: FOXP3, forkhead box P3; NSCLC, non-small cell lung cancer; Tregs, regulatory T cells; IHC, immunohistochemistry; MMP, matrix metalloproteinase; VEGF, vascular endothelial growth factor; EMT, epithelial-mesenchymal transition; ECM, extracellular matrix; T-ALL, T cell acute lymphoblastic leukemia cells; Hes1, Hes family bHLH transcription factor 1; MVD, microvascular density; DAPT, N-(N-(3,5-Difluorophenacetyl)-L-alanyl)-S-phenylglycine t-butyl ester

Key words: forkhead box P3, angiogenic factor, Notch1/Hes1 pathway, epithelial-mesenchymal transition, non-small cell lung cancer and E-cadherin was detected by immunohistochemical (IHC) staining of 55 human NSCLC tissue samples. The results demonstrated that FOXP3 knockdown significantly inhibited the cell migratory and invasive abilities, decreased the concentrations of MMP-2, MMP-9 and VEGF, downregulated the protein expression of vimentin, $\mathrm{N}$-cadherin, Notch1 and Hes family BHLH transcription factor 1 (Hes1), and upregulated the protein expression of E-cadherin. Furthermore, FOXP3 expression was positively associated with $\mathrm{CD} 31^{+}$vascular endothelial cells and negatively correlated with E-cadherin in NSCLC tissues. In addition, the Notch1/Hes1 pathway inhibitor DAPT significantly downregulated the expression of FOXP3 in a dose-dependent manner. Taken together, these findings demonstrated that FOXP3 may facilitate the invasive and migratory abilities of NSCLC cells via regulating the angiogenic factor VEGF, the EMT and the Notch1/Hes1 pathway.

\section{Introduction}

Lung cancer is the most frequent cause of cancer-mortality worldwide (1) and the 5-year survival rate is $<17 \%$ (2). Every year, 1.8 million people are diagnosed with lung cancer and 1.6 million people die of the disease (3). Non-small cell lung cancer (NSCLC) is the most common type of lung cancer and accounts for $\sim 85 \%$ of all cases (4). Despite advances and improvements in surgical treatment for early-stage NSCLC, recurrence and metastasis are still the main factors affecting prognosis (5). The underlying mechanisms of NSCLC metastasis remain poorly understood. It is therefore essential to better characterize the molecular mechanisms associated with NSCLC metastasis, which may contribute to the identification of novel biomarkers and therapeutic targets in NSCLC.

Forkhead box P3 (FOXP3) is a well-known specific marker of regulatory $\mathrm{T}$ cells (Tregs) that has been reported to promote tumour progression due to its immunosuppressive function (6). Previous studies have demonstrated that FOXP3 is expressed in numerous types of cancer cell and has dual roles in carcinogenesis (7-15). Increased expression of FOXP3 in tumour cells is reported to play a protumour role in pancreatic carcinoma (8), 
colorectal cancer (9), thyroid carcinoma (10) and cervical cancer (11), while FOXP3 decreased expression and antitumour role are observed in breast cancer (12), prostate cancer (13), ovarian cancer (14) and $\mathrm{T}$ cell acute lymphoblastic leukaemia (T-ALL) (15). Our previous studies have demonstrated that FOXP3 is highly expressed in NSCLC tissues and that its increased expression is associated with lymph node metastasis and advanced Tumor-Node-Metastasis (TNM) stage $(16,17)$. Furthermore, FOXP3 can promote cell proliferation and reduce cell chemosensitivity to anticancer drugs in $\operatorname{NSCLC}(17,18)$. Another study in NSCLC has reported that FOXP3 promotes tumour growth and metastasis by upregulating the expression levels of cyclin D1, transforming growth factor $\beta 1$, interleukin 35 and heme oxygenase-1 (19). A recent study reported that FOXP3 can activate the Wnt signaling pathway to promote cell proliferation and invasion and the induction of epithelial-mesenchymal transition (EMT) by physically interacting with $\beta$-catenin and transcription factor 4 (TCF4) in NSCLC (20). Although the protumour effect of FOXP3 has been demonstrated in NSCLC, the underlying mechanisms remain to be elucidated.

Tumour cell metastasis is a multistep process involving several crucial events, such as local extracellular matrix (ECM) invasion, tumour angiogenesis, EMT and abnormal activation of signal transduction pathways (21). The Notch1 pathway is a highly conserved signalling pathway that is involved in multiple aspects of cancer biology, including cancer stem cells, angiogenesis and antitumour immunity $(22,23)$. Aberrant activation of this pathway has been reported in many types of cancer and is associated with tumour growth, invasion and metastasis (24). Hes family BHLH transcription factor 1 (Hes1) is the most well-characterized target gene of Notch1, and high levels of Notch1 and Hes1 are associated with a poor prognosis in patients with NSCLC $(25,26)$. However, the correlation between the Notch1/Hes1 pathway and FOXP3 in NSCLC is unclear.

The present study aimed to elucidate the potential mechanisms involving FOXP3 in regulating the metastatic process of NSCLC. The effects of FOXP3 on cell migratory and invasive abilities, vascular endothelial growth factor (VEGF) expression and EMT in NSCLC were explored. The association between FOXP3 and the Notch1/Hes1 pathway was also investigated. The findings from the present study may provide a novel potential therapeutic target in NSCLC.

\section{Materials and methods}

Cell culture. The human NSCLC cell line A549 was obtained from the American Type Culture Collection. Cells were cultured in complete Dulbecco's modified Eagle's medium (DMEM; HyClone; GE Healthcare Life Sciences) supplemented with 10\% FBS (Gibco; Thermo Fisher Scientific, Inc.) and placed at $37^{\circ} \mathrm{C}$ in a humidified incubator containing $5 \% \mathrm{CO}_{2}$

FOXP3 siRNA transfection. Small interfering (si)RNA oligonucleotides specific to FOXP3 (si-FOXP3-1 and si-FOXP3-2) and a negative control siRNA (si-NC) were synthesized by Guangzhou RiboBio Co., Ltd. The sequences of the siRNAs were as follows: si-FOXP3-1, 5'-CAUGGACUACUUCAA GUUCdTdT-3'; si-FOXP3-2, 5'-GAGAGAUGGUACAGU CUCUdTdT-3'; and si-NC, 5'-UUCUCCGAACGUGUC ACGUdTdT-3'. A549 cells $\left(2.5 \times 10^{5}\right)$ were transfected with
si-NC or si-FOXP3-1 or 2 (10 nM final concentration) using Lipofectamine $^{\mathrm{TM}} 2000$ (cat. no. 11668019; Invitrogen; Thermo Fisher Scientific, Inc.) following the manufacturers' protocol. After $6 \mathrm{~h}$, the transfection medium was replaced with DMEM containing 10\% FBS. Transfected cells were eventually harvested at $48 \mathrm{~h}$ for reverse transcription quantitative (RT-q) PCR or at $72 \mathrm{~h}$ for western blotting.

$R T-q P C R$. Total RNA was isolated from cells using the RNAiso Plus Kit (Takara Bio, Inc.) and cDNA was synthesized using the PrimeScript ${ }^{\mathrm{TM}}$ RT Reagent Kit (Takara Bio, Inc.) according to the manufacturers' instructions. Gene expression was detected using SYBR Premix Ex Taq ${ }^{\mathrm{TM}}$ II (Takara Bio, Inc.) according to the manufacturers' protocol on a Piko-Real 24 Real-Time PCR system (Thermo Fisher Scientific, Inc.). The primers for FOXP3, VEGF and GAPDH were synthesized by Sangon Biotech Co., Ltd. The sequences of the primers are shown in Table I. The reaction were performed as follows: $95^{\circ} \mathrm{C}$ for $15 \mathrm{~min}$ and 40 cycles of $95^{\circ} \mathrm{C}$ for $10 \mathrm{sec}$ and $60^{\circ} \mathrm{C}$ for $10 \mathrm{sec}$. The relative gene expression was normalized to endogenous control and expressed as $2^{-\Delta \Delta C q}(27)$.

Western blotting. Cells were washed with ice-cold PBS, lysed with RIPA buffer (Beyotime Institute of Biotechnology) containing a $1 \mathrm{mM}$ protease inhibitor PMSF (Beyotime Institute of Biotechnology) on ice for $30 \mathrm{~min}$ and centrifuged at $15,000 \mathrm{xg}$ for $15 \mathrm{~min}$ at $4^{\circ} \mathrm{C}$. Proteins $(30 \mu \mathrm{g})$ were separated by $10 \%$ SDS-PAGE and transferred onto PVDF membranes. Membranes were blocked with 5\% bovine serum albumin (Sigma-Aldrich; Merck KGaA) for $1 \mathrm{~h}$ at room temperature (RT) and incubated with primary antibodies $(1: 1,000)$ against FOXP3 (cat. no. 4852; Boster Biological Technology), Notch1 (cat. no. 4126; Boster Biological Technology), E-cadherin (cat. no. 0138; Beyotime Institute of Biotechnology), vimentin (cat. no. 0318; Beyotime Institute of Biotechnology), N-cadherin (cat. no. 0243; Beyotime Institute of Biotechnology), Hes1 (cat. no. 2167; Beyotime Institute of Biotechnology) and GAPDH (cat. no. 0006; Beyotime Institute of Biotechnology) overnight at $4^{\circ} \mathrm{C}$. The membranes were then incubated with appropriate horseradish peroxidase (HRP)-conjugated secondary antibodies (1:2,000; cat. no. A0208; cat. no. A0216; Beyotime Institute of Biotechnology) for $1 \mathrm{~h}$ at RT. Enhanced chemiluminescence reagent (BeyoECL Star Kit; Beyotime Institute of Biotechnology) was used to detect the signal on the membrane. The data were analyzed via densitometry using VisionWorksLS 7.1 software (UVP, LLC) and normalized to expression of the internal control GAPDH.

Transwell assay. Cell migratory and invasive abilities were assessed in 24-well Transwell chambers containing inserts of $8.0 \mu \mathrm{m}$ pore size (Corning Inc.). For the invasion assay, the inserts were precoated with $80 \mu 1$ Matrigel (1:8; BD Biosciences). Cells $\left(5 \times 10^{4}\right)$ were suspended in $100 \mu \mathrm{l}$ serum-free DMEM and seeded in the upper chamber of the chamber, whereas $500 \mu \mathrm{l}$ medium containing $10 \%$ FBS was added to the lower chamber to act as a chemoattractant. Following $20 \mathrm{~h}$ incubation, cells on the upper membrane surface were removed and cells that have migrated through the membrane were fixed in $4 \%$ paraformaldehyde for $15 \mathrm{~min}$ at RT and stained with haematoxylin for $5 \mathrm{~min}$ at RT. The number of invaded and migrated cells was counted in five 
Table I. Sequences of the primers used for reverse transcription quantitative PCR.

\begin{tabular}{lcc}
\hline Gene & Primer sequence (5'-3') & Genbank accession no. \\
\hline FOXP3 & & NM_014009.3 \\
Forward & 5'-CACAACATGCGACCCCTTTCACC-3' & \\
Reverse & 5'-AGGTTGTGGCGGATGGCGTTCTTC-3' & \\
VEGF & & NM_001025368.3 \\
Forward & 5'-GTGCCCACTGAGGAGTCCAACATC-3' & \\
Reverse & 5'-GAGCAAGGCCCACAGGGATTTT-3' & \\
GAPDH & & NM_002046.3. \\
Forward & 5'-ATGGGGAAGGTGAAGGTCG-3' \\
Reverse & 5'-GGGTCATTGATGGCAACAATATC-3' & \\
\hline
\end{tabular}

random fields using a light microscope (BX43F; Olympus Corporation) at x400 magnification.

ELISA. Following cell transfection with siRNAs for $72 \mathrm{~h}$, the culture supernatant of A549 cells was harvested and centrifuged at 2,000 x g for $10 \mathrm{~min}$ at RT. The concentrations of matrix metalloproteinase-2 (MMP-2), MMP-9 and VEGF in the culture supernatant were detected using ELISA kits (cat. no. EK0459; cat. no. EK0465; cat. no. EK0539; Boster Biological Technology) according to the manufacturers' protocol. Absorbance was read at $450 \mathrm{~nm}$ on a microplate reader (Tecan Group, Ltd.). The concentrations of detected proteins were calculated according to a standard curve.

Clinical samples. Formalin-fixed paraffin-embedded tissue samples were obtained from 55 patients with NSCLC (average age, 60.5 \pm 9.4 years; 34 men and 21 women) who received treatment at the General Hospital of China National Petroleum Corporation (CNPC) in Jilin between January 2009 and December 2013. Regarding the histological subtype, 35 samples were squamous cell carcinomas and 20 were adenocarcinomas. A total of 24 samples originated from patients who had lymph node metastasis whereas 31 samples were free of metastasis. According to the TNM classification, 27 samples were in stage I, 18 samples were in stage II and 10 samples were in stage III. The inclusion criteria included a clear pathological diagnosis of NSCLC and no prior radiotherapy or chemotherapy. The exclusion criteria included diagnosis with other types of cancer type and a lack of clinicopathological data. This study was approved by the Medical Ethics Committee of CNPC and all patients provided written informed consent.

Immunohistochemical (IHC) staining. Tissue sections were deparaffinized in xylene and rehydrated in a decreasing gradient series of ethanol solutions. Following antigen retrieval and blocking with 3\% hydrogen peroxide for $10 \mathrm{~min}$ at room temperature, sections were incubated with primary antibodies against FOXP3 (1:100; cat. no. 20034; Abcam), CD31 (1:50; cat. no. 53411; Santa) and E-cadherin (ready to use, cat. no. 0092; ZSGB-Bio Co.,Ltd.) overnight at $4^{\circ} \mathrm{C}$. After incubation with an HRP-conjugated secondary antibody (cat. no. PV9000; ZSGB-Bio Co.,Ltd.) for $20 \mathrm{~min}$ at RT, 3,3'-diaminobenzidine was used for chromogen detection.
All sections were evaluated using a double-blinded method. A total of five fields were observed under light microscopy (magnification, $\mathrm{x} 400$ ) and 100 tumour cells were counted in each section. FOXP3 and E-cadherin expression was assessed as previously described (16). CD31-stained sections were evaluated to determine the microvascular density (MVD) and assessed as previously described (28).

Treatment with signalling pathway inhibitors. N-(N(3,5-Difluorophenacetyl)-L-alanyl)-S-phenylglycine t-butyl ester (DAPT; cat. no. SF4139; Beyotime Institute of Biotechnology) was used to inhibit the activity of the Notch1/Hes1 pathway in A549 cells. Briefly, cells $\left(5 \times 10^{5}\right.$ cells per well) in six-well plates were incubated overnight at $37^{\circ} \mathrm{C}$ and treated with or without DAPT (10 or $20 \mu \mathrm{M}$ ) for $48 \mathrm{~h}$. Cells were subsequently collected and used for western blotting.

Statistical analysis. Statistical analysis was conducted using SPSS 17.0 (SPSS Inc.). Data were presented as the means \pm standard deviation. All experiments were performed $\geq$ three times. Differences between groups were analysed using one-way ANOVA followed by Tukey's post hoc test. Pearson correlation analysis was performed to assess the correlation between FOXP3, CD31 and E-cadherin in NSCLC tissues. $\mathrm{P}<0.05$ was considered to indicate a statistically significant difference.

\section{Results}

Efficiency of FOXP3 knockdown in NSCLC cells. Our previous studies have reported that FOXP3 is highly expressed in NSCLC cells $(16,17)$. To investigate the potential function and underlying mechanism of FOXP3 in NSCLC metastasis, two FOXP3-targeted siRNAs (si-FOXP3-1 and si-FOXP3-2) were transfected into A549 cells, and the knockdown efficiency of FOXP3 was measured by RT-qPCR and western blotting. The results demonstrated that the mRNA and protein expression of FOXP3 in the FOXP3-knockdown group was significantly downregulated $(\mathrm{P}<0.01)$, showing an knockdown efficiency of $\sim 60 \%$ (Fig. 1A and B).

FOXP3 knockdown inhibits the migratory and invasive abilities of NSCLC cells. To explore the effects of FOXP3 on the migratory and invasive abilities of NSCLC cells, Transwell 

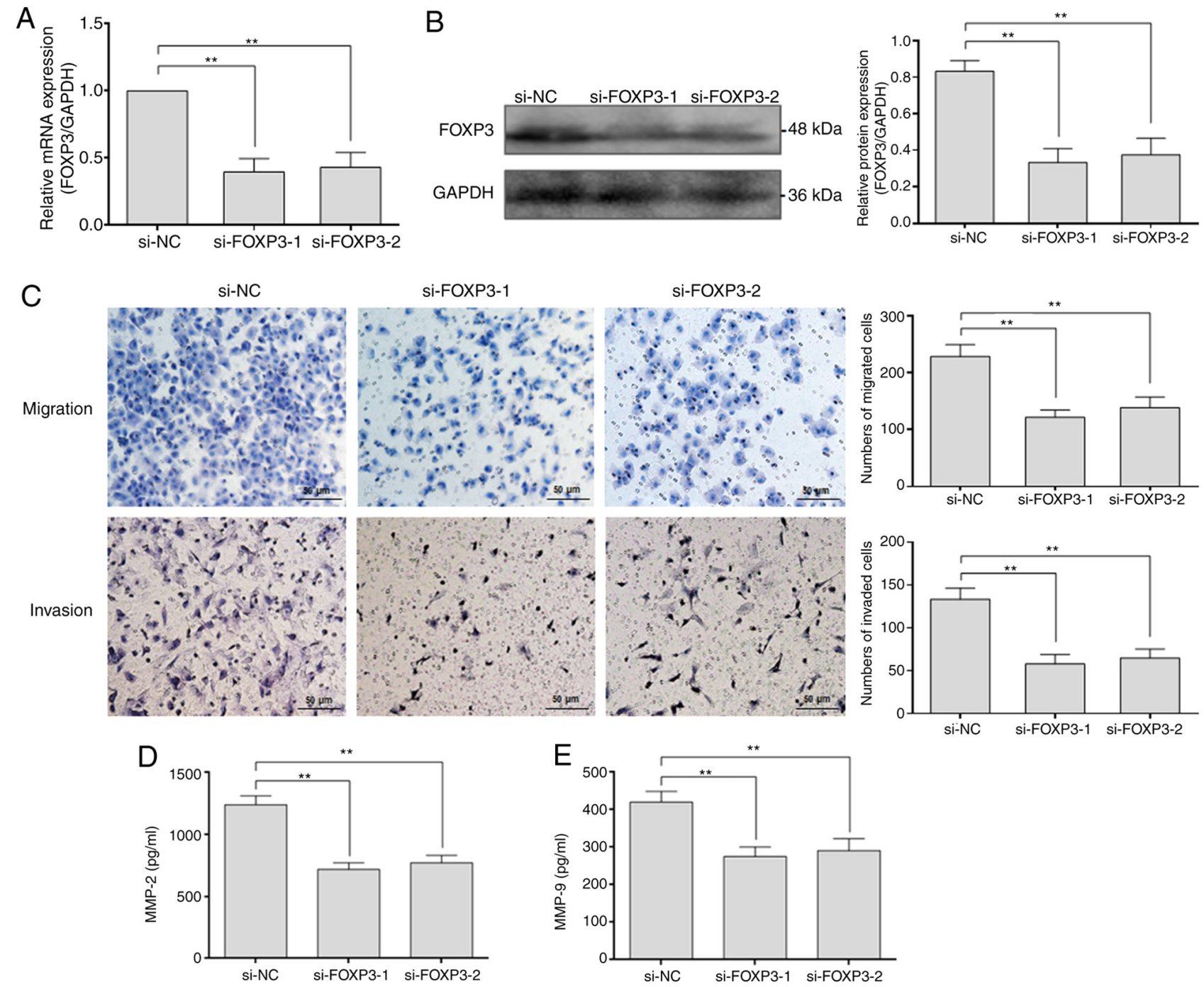

Figure 1. FOXP3 promotes the migratory and invasive abilities of A549 cells. (A) Reverse transcription quantitative PCR assessing the efficacy of FOXP3 knockdown using specific siRNAs in A549 cells. (B) Western blotting assessing the efficacy of FOXP3 knockdown using specific siRNAs in A549 cells. (C) Transwell assays assessing the cell migratory and invasive abilities of A549 following FOXP3 knockdown. (D and E) ELISA detection of MMP-2 and MMP-9 secretion from A549 cells $72 \mathrm{~h}$ following FOXP3 knockdown. ${ }^{* *} \mathrm{P}<0.01$. FOXP3, forkhead box P3; MMP-2, matrix metalloproteinase-2; MMP-9, matrix metalloproteinase-9; si, small interfering; NC, negative control.

assays were performed following FOXP3 knockdown in A549 cells. The results demonstrated that the numbers of migrated and invaded cells in the FOXP3-knockdown group were significantly decreased compared with the si-NC group $(\mathrm{P}<0.01$; Fig. $1 \mathrm{C})$, indicating that FOXP3 may enhance the migratory and invasive abilities of NSCLC cells. Subsequently, the expression of MMP-2 and MMP-9, which are two key molecules involved in cell migration and invasion, was examined. The results from ELISA demonstrated that the concentrations of MMP-2 and MMP-9 in the culture supernatant of FOXP3-knockdown group were significantly decreased compared with those in the culture supernatant of the si-NC group ( $\mathrm{P}<0.01 ;$ Fig. $1 \mathrm{D}$ and $\mathrm{E})$. These data indicated that FOXP3 may promote the migratory and invasive abilities of NSCLC cells by increasing MMP-2 and MMP-9 secretion.

FOXP3 regulates the expression of the angiogenic factor $V E G F$ in NSCLC. To determine the correlation between FOXP3 expression and MVD, the expression of FOXP3 and the endothelial cell marker CD31 was detected by IHC staining in 55 NSCLC tissue samples. The results demonstrated that increased expression of FOXP3 was mostly observed in samples with a higher MVD-CD31 rather than a lower MVD-CD31 (Fig. 2A). Pearson's correlation analysis showed a positive correlation between FOXP3 expression and MVD-CD31 ( $\mathrm{r}=0.326 ; \mathrm{P}=0.015$; Table II), suggesting that FOXP3 may play a role in blood vessel formation in NSCLC tissues. Because VEGF is one of the most important angiogenic factors, we further examined the expression of VEGF by RT-qPCR and ELISA following FOXP3 knockdown in A549 cells. The expression of VEGF at the mRNA and protein levels was significantly decreased in the FOXP3-knockdown groups compared with the si-NC group $(\mathrm{P}<0.01$; Fig. $2 \mathrm{~B}$ and $\mathrm{C})$, demonstrating that FOXP3 may regulate the expression of the angiogenic factor VEGF in NSCLC cells.

FOXP3 induces EMT in NSCLC. EMT is a key process in NSCLC invasion and metastasis. To investigate whether 
A

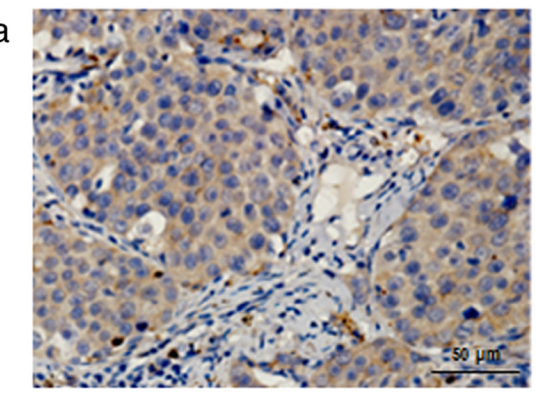

C
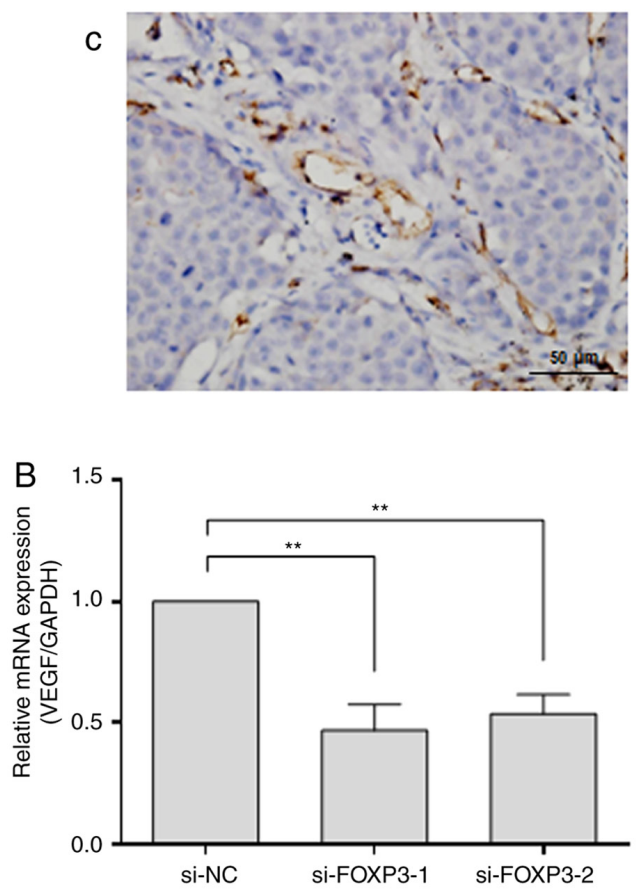
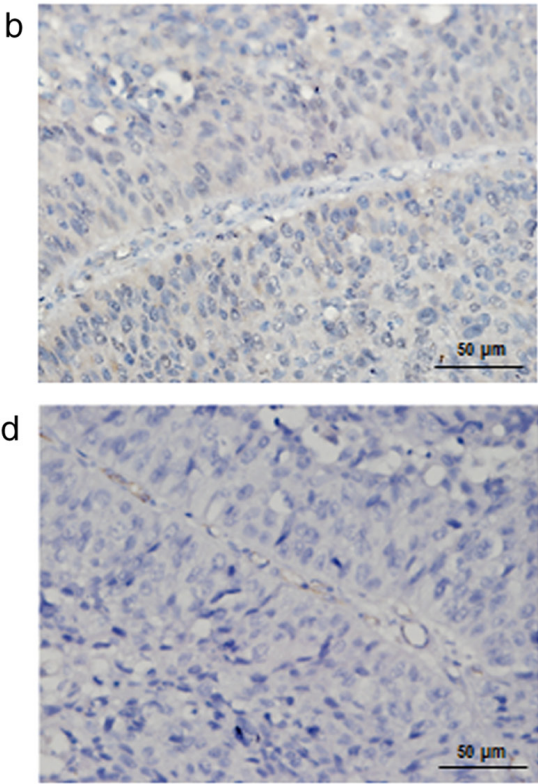

C

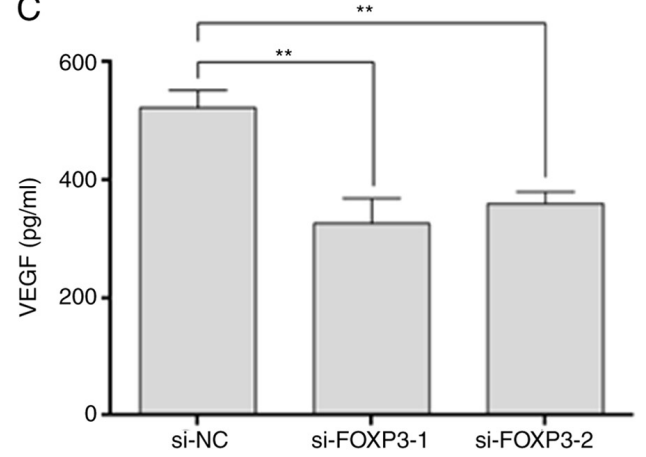

Figure 2. Expression of FOXP3 and CD31 in NSCLC tissues and regulation of VEGF expression by FOXP3 in A549 cells. (A) FOXP3 expression was positively associated with CD $31^{+}$vascular endothelial cells in individual NSCLC tissue samples according to immunohistochemistry staining (magnification, $\mathrm{x} 400$ ). $\mathrm{a}$ and $\mathrm{c}$ show positive expression of FOXP3 and CD31; $\mathrm{b}$ and $\mathrm{d}$ show negative expression of FOXP3 and CD31. (B) Expression levels of VEGF were detected by reverse transcription quantitative PCR $48 \mathrm{~h}$ following FOXP3 knockdown in A549 cells. (C) Expression levels of VEGF were detected by ELISA assay $72 \mathrm{~h}$ following FOXP3 knockdown in A549 cells. ${ }^{* *} \mathrm{P}<0.01$. FOXP3, forkhead box P3; VEGF, vascular endothelial growth factor; NSCLC, non-small cell lung cancer; si, small interfering; NC, negative control.

FOXP3-mediated metastasis is associated with EMT process, the epithelial marker E-cadherin was detected in NSCLC tissue samples, and the correlation between FOXP3 and E-cadherin expression was analysed. The results from IHC staining demonstrated that FOXP3 expression was significantly increased, while E-cadherin expression was significantly decreased in NSCLC tissues (Fig. 3A). A negative correlation was reported between FOXP3 and E-cadherin expression in the NSCLC tissue samples ( $r=-0.297 ; \mathrm{P}=0.028$; Table III), suggesting that FOXP3-mediated metastasis may involve EMT process. To confirm the relationship between FOXP3 and EMT, the expression of the EMT-related proteins vimentin, $\mathrm{N}$-cadherin and E-cadherin was assessed by western blotting following FOXP3 knockdown in A549 cells. The results demonstrated that FOXP3 knockdown significantly decreased the expression of vimentin and $\mathrm{N}$-cadherin but significantly decreased that of E-cadherin ( $\mathrm{P}<0.01$; Fig. 3B), indicating that FOXP3 knockdown may reverse EMT in NSCLC cells.

Link between FOXP3 and the Notch1/Hesl pathway in NSCLC cells. The Notch1/Hes1 pathway is a vital signalling pathway in cancer pathogenesis. To investigate whether the promotion of
NSCLC metastasis by FOXP3 might involve the Notch1/Hes1 pathway, the expression of Notch1 and Hes1 was determined by western blotting following FOXP3 knockdown in A549 cells. The results demonstrated that the expression of Notch1 and Hes1 in the FOXP3-knockdown group was significantly decreased compared with that in the si-NC group $(\mathrm{P}<0.01$; Fig. 4A). Subsequently, the effects of Notch1/Hes1 pathway blockade on the expression of FOXP3 in A549 cells were detected by western blotting. Following treatment with the Notch1 pathway inhibitor DAPT $(0,10$ or $20 \mu \mathrm{M})$, the expression of Notch1, Hes1 and FOXP3 was significantly decreased in a dose-dependent manner $(\mathrm{P}<0.01$; Fig. 4B). These findings suggested that the pro-metastatic effect of FOXP3 may be closely linked to the Notch1/Hes1 pathway in NSCLC cells.

\section{Discussion}

Previous studies from our laboratory investigated the expression of FOXP3 in clinical NSCLC tissue samples and its association with clinicopathological characteristics of patients with NSCLC $(16,17)$. The results demonstrated that 
Table II. Correlation analysis between FOXP3 and MVD-CD31 in non-small cell lung cancer tissues.

\begin{tabular}{lccccc}
\hline & \multicolumn{2}{c}{ FOXP3 expression } & & & \\
\cline { 2 - 4 } MVD-CD31 expression & Positive & Negative & Total & r & P-value \\
\hline High & 24 & 7 & 31 & 0.326 & $0.015^{\text {a }}$ \\
Low & 11 & 13 & 24 & & \\
Total & 35 & 20 & 55 & & \\
\hline
\end{tabular}

${ }^{a} \mathrm{P}<0.05$. FOXP3, forkhead box P3; MVD, microvascular density.

A a

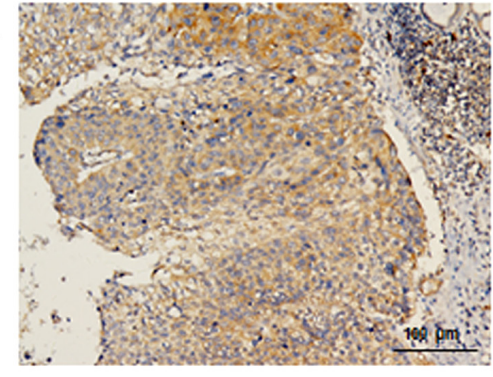

c

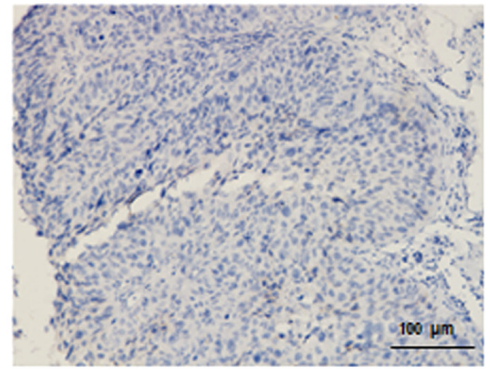

B

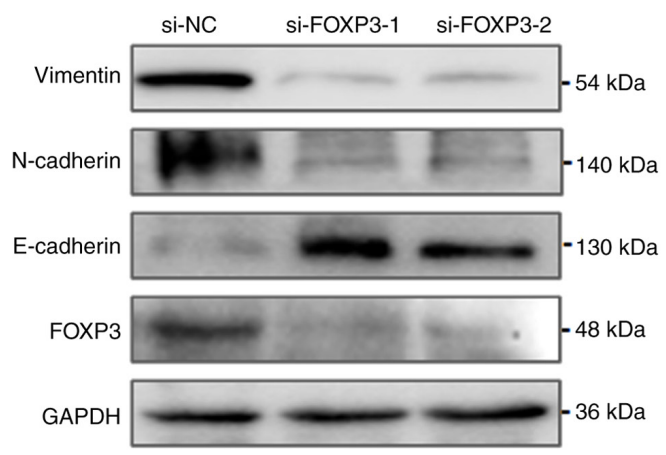

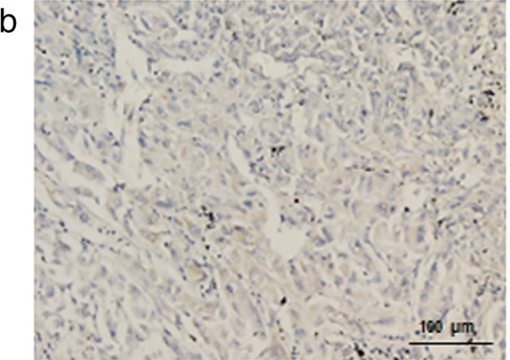

d

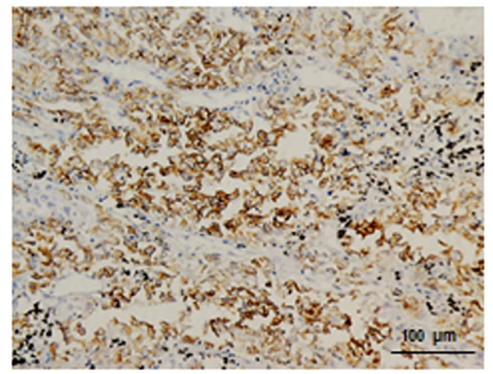

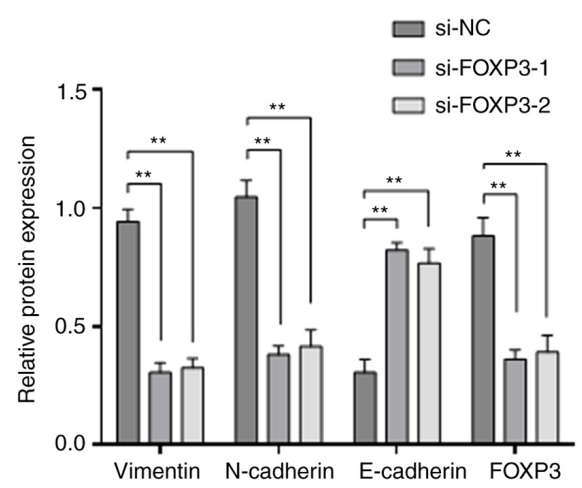

Figure 3. FOXP3 induces epithelial-to-mesenchymal transition in NSCLC tissues and cells. (A) FOXP3 expression was inversely associated with E-cadherin expression in NSCLC tissue samples according to immunohistochemistry staining (magnification, x200). (a and c) show positive expression of FOXP3 and negative expression of E-cadherin; ( $b$ and d) show negative expression of FOXP3 and positive expression of E-cadherin. (B) Expression of vimentin, N-cadherin and E-cadherin was examined by western blotting $72 \mathrm{~h}$ after FOXP3 knockdown in A549 cells. ${ }^{* *} \mathrm{P}<0.01$. FOXP3, forkhead box P3; NSCLC, non-small cell lung cancer; si, small interfering; NC, negative control.

FOXP3 expression is elevated in squamous cell carcinoma and adenocarcinoma compared with normal lung tissues and positively correlated with lymph node metastasis and TNM stage in patients with NSCLC patients, indicating that FOXP3 may serve crucial roles in NSCLC metastasis and progression $(16,17)$. In the present study, the molecular mechanisms by which FOXP3 can promote metastasis in NSCLC tissues and cells were investigated.
Tumour metastasis is characterized by tumour cells acquiring the ability to migrate and invade from the primary tumour to distant organs. In the present study, the migratory and invasive abilities of A549 cells were significantly inhibited following FOXP3 knockdown according to Transwell assays. Combined with our previous studies $(16,17)$, the results from the present study further demonstrated that NSCLC cells with high FOXP3 expression may have a relatively high metastatic 
Table III. Correlation analysis between FOXP3 and E-cadherin in non-small cell lung cancer tissues.

\begin{tabular}{lccccc}
\hline & \multicolumn{2}{c}{ FOXP3 expression } & & & \\
\cline { 2 - 5 } E-cadherin expression & Positive & Negative & Total & r & P-value \\
\hline Positive & 12 & 13 & 25 & -0.297 & $0.028^{\text {a }}$ \\
Negative & 23 & 7 & 30 & & \\
Total & 35 & 20 & 55 & & \\
\hline
\end{tabular}

${ }^{\mathrm{a}} \mathrm{P}<0.05$. FOXP3, forkhead box P3.

A

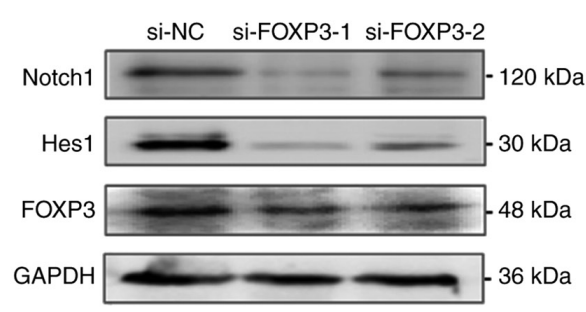

B

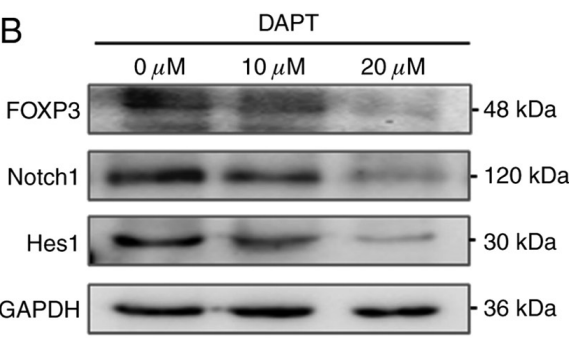

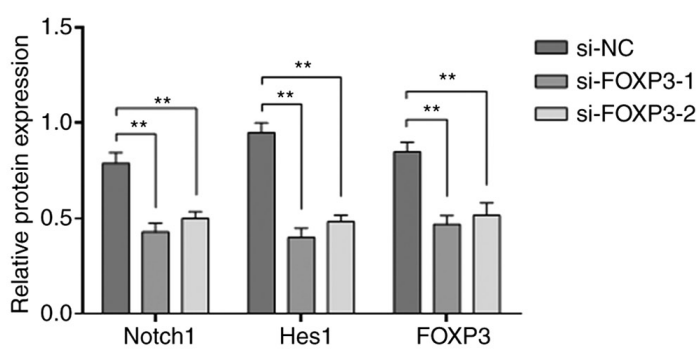

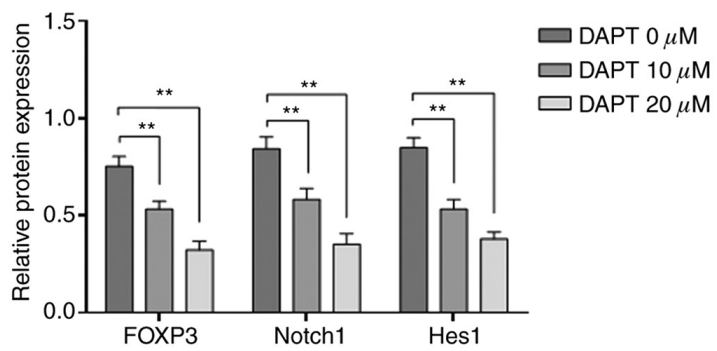

Figure 4. Association between FOXP3 and Notch1/Hes1 pathway in A549 cells. (A) Expression Notch1 and Hes1 was examined by western blotting assay 72 h after FOXP3 knockdown in A549 cells. (B) Expression of FOXP3 was examined by western blotting analysis $48 \mathrm{~h}$ after treatment with DAPT at 10 and $20 \mu \mathrm{M}$. ${ }^{* *} \mathrm{P}<0.01$. FOXP3, forkhead box P3; Hes1, Hes family bHLH transcription factor 1; DAPT, N-(N-(3,5-difluorophenacetyl)-L-alanyl)-S-phenylglycine t-butyl ester; si, small interfering; NC, negative control.

potential. The degradation of the ECM by MMPs is an essential initial step in tumour invasion and metastasis (29). MMP-2 and MMP-9, which are both type IV collagenases, have been shown to play important roles in cell migration, invasion, angiogenesis and metastasis of malignant tumours $(30,31)$. A previous study reported that FOXP3 upregulation could decrease cell migration, invasion and MMP-2 expression in epithelial ovarian cancer (14). In the present study, FOXP3 knockdown significantly inhibited the secretion of MMP-2 and MMP-9 by A549 cells, which suggested that FOXP3 may promote the motility of NSCLC cells by increasing MMP-2 and MMP-9 secretion.

High MVD and increased expression of angiogenic factors may increase tumour invasive and metastatic abilities (32). In the present study, MVD was assessed using CD31-positive vascular endothelial cells. The results demonstrated that NSCLC tissue samples with high FOXP3 expression had a higher MVD-CD31 than those with low FOXP3 expression, and statistical analysis showed a strong correlation between FOXP3 and MVD-CD31, indicating that FOXP3 may play a role in blood vessel formation in NSCLC tissues. VEGF is one of the most important angiogenic factors that can stimulate the proliferation of vascular endothelial cells, and increasing the permeability of the vessels and blocking the VEGF pathway can suppress tumour angiogenesis and metastasis (33). A previous study reported that FOXP3 expression is positively correlated with VEGF-C which is a specific regulatory factor of endothelial cells of blood and lymphatic vessels in cervical cancer tissues (34). Conversely, a study reported that FOXP3 could inhibit angiogenesis by downregulating VEGF in breast cancer (35). However, whether FOXP3 regulates VEGF in NSCLC remains unclear. In the present study, FOXP3 knockdown significantly decreased VEGF mRNA and protein expression in A549 cells, which indicated that FOXP3 may upregulate VEGF expression and subsequently facilitate the invasion and metastasis of NSCLC cells.

EMT is a key event in local invasion and distant metastasis in human cancers, and this transformation is characterized by epithelial cells losing epithelial cell markers, such as the cell adhesion molecule E-cadherin, and acquiring a mesenchymal phenotype, characterized by vimentin and N-cadherin expression (36). Previous studies demonstrated that cancer cells with EMT phenotype might exhibit highly invasive and metastatic abilities that lead to an elevated rate of distant metastases and 
a worse prognosis in patients with cancer, including breast, colorectal and lung cancer (37-39). Loss or downregulation of E-cadherin expression is a major hallmark of EMT, which results in reduced cell-cell connections and enhanced cellular mobility. In the present study, the correlation between the expression of FOXP3 and E-cadherin in NSCLC tissues was analysed. The results revealed a negative correlation between the two proteins, indicating that FOXP3-mediated metastases may involve EMT process in NSCLC. This hypothesis was further confirmed by results showing that FOXP3 knockdown reversed the EMT phenotype in A549 cells. This finding was supported by a previous study from Yang et al (20) who demonstrated that FOXP3 could enhance the function of $\beta$-catenin and TCF4 in order to activate EMT-related molecules, such as snail and slug, leading to the induction of EMT in human NSCLC cell lines. The results on NSCLC tissues and cells from the present study further confirmed that FOXP3 might facilitate the invasion and metastasis of NSCLC cells via EMT induction.

The Notch1/Hes1 pathway plays a critical role in tumorigenesis and promotes angiogenesis and EMT in many types of malignant tumour, including NSCLC $(24,40,41)$. It has been reported that blocking the Notch1 pathway can downregulate FOXP3 expression in T-ALL and melanoma cells $(15,42)$. Combined with the aforementioned results and the pro-metastatic effect of FOXP3 demonstrated in the present study, we hypothesized that FOXP3 and the Notch1/Hes1 pathway may be closely related in NSCLC. This hypothesis was confirmed by results showing that FOXP3 knockdown significantly decreased the activity of the Notch1/Hes1 pathway and that Notch1/Hes1 pathway blockade significantly downregulated the expression of FOXP3 in a dose-dependent manner. These findings therefore suggested a possible interaction between FOXP3 and the Notch1/Hes1 pathway and revealed that the pro-metastatic effect of FOXP3 may be linked to the Notch1/Hes1 pathway in NSCLC cells.

This study presented some limitations. Firstly, this study was performed using only one cell line. Secondly, the molecular mechanism underlying the interaction between FOXP3 and the Notch1 pathway in NSCLC progression was not determined and requires further investigation.

In summary, the results from the present study demonstrated that FOXP3 may facilitate the invasion and metastasis of NSCLC cells via regulating VEGF, EMT and the Notch1/Hes1 pathway. This study provided novel mechanistic insights into the role of FOXP3 in NSCLC metastasis and suggested that FOXP3 may be considered as a promising therapeutic target in NSCLC.

\section{Acknowledgements}

Not applicable.

\section{Funding}

This research was supported by the Technology Research Projects of the Education Department of Jilin Province (grant no. JJKH20180353KJ), the Health Department of Jilin Province (grant no. 2020J017) and the Science and Technology Department of Jilin Province (grant nos. 20190201220JC and YDZJ202101ZYTS089).

\section{Availability of data and materials}

The datasets used and/or analysed during the current study are available from the corresponding author on reasonable request.

\section{Authors' contributions}

CL, HW, HF, CH, YP and XG performed the experiments. CL and $\mathrm{XG}$ designed the experiments. CL and XG confirm the authenticity of all the raw data. All authors read and approved the final manuscript.

\section{Ethics approval and consent to participate}

The present study was approved by the Ethics Committee of the General Hospital of China National Petroleum Corporation (approval no. 2018031). Patients or their family members were fully informed of the study details and provided written informed consent.

\section{Patient consent for publication}

Not applicable.

\section{Competing interests}

The authors declare that they have no competing interests.

\section{References}

1. Torre LA, Siegel RL and Jemal A: Lung cancer statistics. Adv Exp Med Biol 893: 1-19, 2016.

2. Hirsch FR, Scagliotti GV, Mulshine JL, Kwon R, Curran WJ Jr, Wu YL and Paz-Ares L: Lung cancer: Current therapies and new targeted treatments. Lancet 389: 299-311, 2017.

3. Ferlay J, Soerjomataram I, Dikshit R, Eser S, Mathers C, Rebelo M, Parkin DM, Forman D and Bray F: Cancer incidence and mortality worldwide: Sources, methods and major patterns in GLOBOCAN 2012. Int J Cancer 136: E359-E386, 2015.

4. Souza MC, Cruz OG and Vasconcelos AG: Factors Associated with disease-specific survival of patients with non-small cell lung cancer. J Bras Pneumol 42: 317-325, 2016 (In English, Portuguese).

5. Zinner R, Visseren-Grul C, Spigel DR and Obasaju C: Pemetrexed clinical studies in performance status 2 patients with non-small cell lung cancer (Review). Int J Oncol 48: 13-27, 2016.

6. Togashi Y, Shitara K and Nishikawa H: Regulatory T cells in cancer immunosuppression-implications for anticancer therapy. Nat Rev Clin Oncol 16: 356-371, 2019.

7. Triulzi T, Tagliabue E, Balsari A and Casalini P: FOXP3 expression in tumor cells and implications for cancer progression. J Cell Physiol 228: 30-35, 2013.

8. Hinz S, Pagerols-Raluy L, Oberg HH, Ammerpohl O, Grüssel S, Sipos B, Grützmann R, Pilarsky C, Ungefroren H, Saeger HD, et al: Foxp3 expression in pancreatic carcinoma cells as a noveral mechanism of immune evasion in cancer. Cancer Res 67: 8344-8350, 2007.

9. Kim M, Grimmig T, Grimm M, Lazariotou M, Meier E, Rosenwald A, Tsaur I, Blaheta R, Heemann U, Germer CT, et al: Expression of Foxp3 in colorectal cancer but not in Treg cells correlates with disease progression in patients with colorectal cancer. PLoS One 8: e53630, 2013.

10. Chu R, Liu SY, Vlantis AC, van Hasselt CA, Ng EK, Fan MD, $\mathrm{Ng}$ SK, Chan AB, Du J, Wei W, et al: Inhibition of Foxp3 in cancer cells induces apoptosis of thyroid cancer cells. Mol Cell Endocrinol 399: 228-234, 2015.

11. Zeng C, Yao Y, Jie W, Zhang M, Hu X, Zhao Y, Wang S, Yin J and Song Y: Up-regulation of Foxp3 participates in progression of cervical cancer. Cancer Immunol Immunother 62: 481-487, 2013. 
12. Zuo T, Wang L, Morrison C, Chang X, Zhang H, Li W, Liu Y, Wang Y, Liu X, Chan MW, et al: FOXP3 is an X-linked breast cancer suppressor gene and an important repressor of the HER-2/ErbB2 oncogene. Cell 129: 1275-1286, 2007.

13. Wang L, Liu R, Li W, Chen C, Katoh H, Chen GY, McNally B, Lin L, Zhou P, Zuo T, et al: Somatic single hits inactivate the $\mathrm{X}$-linked tumor suppressor FOXP3 in the prostate. Cancer Cell 16: 336-346, 2009.

14. Zhang HY and Sun H: Up-regulation of Foxp3 inhibits cell proliferation, migration and invasion in epithelial ovarian cancer. Cancer Lett 287: 91-97, 2010.

15. Luo X, Tan H, Zhou Y, Xiao T, Wang C and Li Y: Notch1 signaling is involved in regulating Foxp3 expression in T-ALL. Cancer Cell Int 13: 34, 2013

16. Fu HY, Li C, Yang W, Gai XD, Jia T, Lei YM and Li Y: FOXP3 and TLR4 protein expression are correlated in non-small cell lung cancer: Implications for tumor progression and escape. Acta Histochem 115: 151-157, 2013.

17. Li C, Sun L, Jiang R, Wang P, Xue H, Zhan Y and Gai X Downregulation of FOXP3 inhibits cell proliferation and enhances chemosensitivity to cisplatin in human lung adenocarcinoma. Pathol Res Pract 213: 1251-1256, 2017.

18. Li C, Yang W, Gai X, Zhang Y, Li Y and Fu H: Foxp3 overexpression decreases sensitivity to chemotherapy in mouse Lewis lung cancer cells. Mol Med Rep 6: 977-982, 2012.

19. Li Y, Li D, Yang W, Fu H, Liu Y and Li Y: Overexpression of the transcription factor FOXP3 in lung adenocarcinoma sustains malignant character by promoting G1/S transition gene CCND1. Tumour Biol 37: 7395-7404, 2016.

20. Yang S, Liu Y, Li MY, Ng CSH, Yang SL, Wang S, Zou C, Dong Y, Du J, Long X, et al: FOXP3 promotes tumor growth and metastasis by activating Wnt/ $\beta$-catenin signaling pathway and EMT in non-small cell lung cancer. Mol Cancer 16: 124, 2017.

21. Valastyan S and Weinberg RA: Tumor metastasis: Molecular insights and evolving paradigms. Cell 147: 275-292, 2011.

22. Takebe N, Miele L, Harris PJ, Jeong W, Bando H, Kahn M, Yang SX and Ivy SP: Targeting notch Hedgehog, and Wnt pathways in cancer stem cells: Clinical update. Nat Rev Clin Oncol 12: 445-464, 2015.

23. Yang Z, Qi Y, Lai N, Zhang J, Chen Z, Liu M, Zhang W, Luo R and Kang S: Notch1 signaling in melanoma cells promoted tumor-induced immunosuppression via upregulation of TGF- $\beta 1$ J Exp Clin Cancer Res 37: 1, 2018.

24. Teoh SL and Das S: Notch signalling pathways and their importance in the treatment of cancers. Curr Drug Targets 19: 128-143, 2018.

25. Zou B, Zhou XL, Lai SQ and Liu JC: Notch signaling and non-small cell lung cancer. Oncol Lett 15: 3415-3421, 2018.

26. Yuan X, Wu H, Xu H, Han N, Chu Q, Yu S, Chen Y and Wu K Meta-analysis reveals the correlation of Notch signaling with non-small cell lung cancer progression and prognosis. Sci Rep 5: $10338,2015$.

27. Livak KJ and Schmittgen TD: Analysis of relative gene expression data using real-time quantitative PCR and the 2 (-Delta DeltaC (T)) method. Methods 25: 402-408, 2001.

28. Li C, Ma X, Tan C, Fang H, Sun Y and Gai X: IL-17F expression correlates with clinicopathologic factors and biological markers in non-small cell lung cancer. Pathol Res Pract 215: 152562, 2019.
29. Tang YQ, Jaganath IB, Manikam R and Sekaran SD: Phyllanthus spp. exerts anti-angiogenic and anti-metastatic effects through inhibition on matrix metalloproteinase enzymes. Nutr Cancer 67: 783-795, 2015

30. Pittayapruek P, Meephansan J, Prapapan O, Komine M and Ohtsuki M: Role of matrix metalloproteinases in photoaging and photocarcinogenesis. Int J Mol Sci 17: 868, 2016.

31. Zhao L, Niu H, Liu Y, Wang L, Zhang N, Zhang G, Liu R and Han M: LOX inhibition downregulates MMP-2 and MMP-9 in gastric cancer tissues and cells. J Cancer 10: 6481-6490, 2019.

32. Giatromanolaki A, Lyberakidis G, Lyratzopoulos N, Koukourakis MI, Sivridis E and Manolas C: Angiogenesis and angiogenic factor expression in thyroid cancer. J BUON 15: $357-361,2010$.

33. Sadremomtaz A, Kobarfard F, Mansouri K, Mirzanejad L and Asghari SM: Suppression of migratory and metastatic pathways via blocking VEGFR1 and VEGFR2. J Recept Signal Transduct Res 38: 432-441, 2018.

34. Tang J, Yang Z, Wang Z, Li Z, Li H, Yin J, Deng M, Zhu W and Zeng C: Foxp3 is correlated with VEGF-C expression and lymphangiogenesis in cervical cancer. World J Surg Oncol 15 $173,2017$.

35. Li X, Gao Y, Li J, Zhang K, Han J, Li W, Hao Q, Zhang W, Wang S, Zeng C, et al: FOXP3 inhibits angiogenesis by downregulating VEGF in breast cancer. Cell Death Dis 9: 744, 2018.

36. Tsubakihara Y and Moustakas A: Epithelial-mesenchymal transition and metastasis under the control of transforming growth factor beta. Int J Mol Sci 19: 3672, 2018.

37. Li P, Sun T, Yuan Q, Pan G, Zhang J and Sun D: The expressions of NEDD9 and E-cadherin correlate with metastasis and poor prognosis in triple-negative breast cancer patients. Onco Targets Ther 9: 5751-5759, 2016.

38. He X, Chen Z, Jia M and Zhao X: Downregulated E-cadherin expression indicates worse prognosis in Asian patients with colorectal cancer: Evidence from meta-analysis. PLoS One 8: e70858, 2013

39. Ancel J, Dewolf M, Deslée G, Nawrocky-Raby B, Dalstein V, Gilles C and Polette M: Clinical impact of the epithelial-mesenchymal transition in lung cancer as a biomarker assisting in therapeutic decisions. Cells Tissues Organs 1-19, 2020 (Epub ahead of print)

40. Yang MH, Zang YS, Huang H, Chen K, Li B, Sun GY and Zhao XW: Arsenic trioxide exerts anti-lung cancer activity by inhibiting angiogenesis. Curr Cancer Drug Targets 14: 557-566, 2014.

41. Gao YP, Li Y, Li HJ and Zhao B: LncRNA NBR2 inhibits EMT progression by regulating Notch1 pathway in NSCLC. Eur Rev Med Pharmacol Sci 23: 7950-7958, 2019.

42. Skarmoutsou E, Bevelacqua V, D' Amico F, Russo A, Spandidos DA, Scalisi A, Malaponte G and Guarneri C: FOXP3 expression is modulated by TGF- $\beta 1 / \mathrm{NOTCH} 1$ pathway in human melanoma. Int J Mol Med 42: 392-404, 2018.

This work is licensed under a Creative Commons Attribution-NonCommercial-NoDerivatives 4.0 International (CC BY-NC-ND 4.0) License. 